\title{
STOCHASTIC CROSS-LAYER RESOURCE ALLOCATION FOR WIRELESS NETWORKS USING ORTHOGONAL ACCESS: OPTIMALITY AND DELAY ANALYSIS
}

\author{
Antonio G. Marques* Georgios B. Giannakis ${ }^{\dagger} \quad$ Javier Ramos* \\ *Dept. of Signal Theory and Communications, Rey Juan Carlos University, Madrid, Spain \\ ${ }^{\dagger}$ Dept. of Electrical Engineering, University of Minnesota, Minneapolis, MN, USA
}

\begin{abstract}
Efficient design of wireless networks requires implementation of cross-layer algorithms that exploit channel state information. Capitalizing on convex optimization and stochastic approximation tools, this paper develops a stochastic algorithm that allocates resources at network, link, and physical layers so that a sum-utility of the average end-to-end rates is maximized. Focus is placed on networks where interference is strong and nodes transmit orthogonally over a set of parallel channels. Convergence of the developed stochastic schemes is characterized, and the average queue delays are obtained in closed form.
\end{abstract}

Index Terms - Resource management, cross-layer design, stochastic approximation, delay effects.

\section{INTRODUCTION}

Non-linear optimization has been successfully used to analyze and design cross-layer algorithms for wireless networks; see e.g., [2] and references therein. The optimal design of the network is obtained by formulating a constrained optimization problem that involves variables from different layers and exploits information about the fading channel. The solution of this optimization problem dictates how resources of different layers have to be allocated, while the structure of the solution typically indicates how the signalling protocols have to be designed.

In this context, the present paper aims to optimally design a wireless network whose operating conditions are the following. At the network layer, nodes receive packets from different applications, which entail different utility levels [6]. At the link layer, nodes access orthogonally a set of parallel flat fading channels. Orthogonal here means that if a terminal is transmitting, no other link interfering with that transmission can be active [4], [6]. At the physical layer, nodes can adapt their power and rate loadings in every channel.

The optimization problem is formulated as a sum-utility maximization that involves variables averaged over all possible states of the fading channel. The optimal cross-layer

The work in this paper was supported by the USDoD ARO grant No. W911NF-05-1-0283 and by the C. A. Madrid grant No. P-TIC-000223-0505; and also through collaborative participation in the CNC sponsored by the U.S. ARL under the CTA Program, Cooperative Agreement DAAD19-01-20011. The U.S. Government is authorized to reproduce and distribute reprints for Government purposes notwithstanding any copyright notation thereon. resource allocation turns out to be a function of the instantaneous channel state information (CSI) and the optimum Lagrange multipliers associated with the optimization problem. Using stochastic approximation tools [3], online schemes to estimate the value of the multipliers are proposed. Convergence and optimality of the stochastic schemes is characterized. As in [4], by carefully designing the stochastic schemes, relationships between the Lagrange multipliers and the queues are established. Such relationships allow one to characterize the stability and the average queue delay of the developed schemes.

Section 2 introduces notation, describes the operation of the different layers, and formulates the optimization problem that will give rise to the optimal resource allocation. The optimum solution is presented in Section 3. Stochastic algorithms together with their convergence analysis are presented in Section 4. Finally, the stability and the average queue delay of the novel stochastic schemes are analyzed in Section 5. Due to space limitation, numerical examples illustrating the theoretical claims of this paper are not included but can be temporally found in [9] and later on in the journal version of this conference paper. ${ }^{1}$

\section{PROBLEM STATEMENT}

Consider a multi-hop wireless network with $I$ nodes, such that each node $i$ is physically linked with all other nodes $j \neq i$ in the network. Although this represents a worst-case scenario from an interference perspective, it simplifies scheduling. Nodes can transmit orthogonally over a set of $K$ flatfading parallel channels. The $k$ th channel's instantaneous power gain from node $i$ to node $j$ is denoted by $h_{i j}^{k}$; and represents the noise-normalized squared magnitude of the fading coefficient. The overall CSI is described by the random vector $\mathbf{h}$ that collects all $h_{i j}^{k}$ gains. Channels are assumed to be ergodic and are allowed to be correlated.

For this network, we wish to develop adaptive algorithms that use the instantaneous CSI to allocate resources at the network, link, and physical layers so that pre-specified QoS metrics are optimized. Next, we describe the operation of each layer.

\footnotetext{
${ }^{1}$ Notation: $|\mathcal{X}|$ denotes the cardinality of the set $\mathcal{X} ; x^{*}$ the optimal value of variable $x ; \mathbb{1}_{\{\cdot\}}$ the indicator function $\left(\mathbb{1}_{\{x\}}=1\right.$ if $x$ is true and zero otherwise); and $[x]_{a}^{b}$ the projection of $x$ onto the $[a, b]$ interval, i.e., $[x]_{a}^{b}=\min \{\max \{a, x\}, b\}$. Finally, for a function $f(\cdot),(f)^{-1}(\cdot)$ denotes its inverse and $\dot{f}(\cdot)$ its derivative.
} 
Network layer operation: Packets generated exogenously at each node correspond to possibly different applications (such as video, voice, or file transfer), and are destined for different sink nodes. Packet streams will be refereed as flows and will be indexed by $f$. Each node serves flows that have other nodes as destination. The destination node associated with a flow $f$ is denoted by $d(f)$, while the average arrival rate of exogenous packets of flow $f$ to node $i$ is denoted by $\bar{a}_{i}^{f}$. The instantaneous rate of flow $f$ that during the channel realization $\mathbf{h}$ is routed from node $i$ to node $j$ is denoted by $r_{i j}^{f}(\mathbf{h})$. As customary in communication systems, we assume that nodes are equipped with queues (buffers) capable of storing the incoming packets. For such queues to be stable, the following necessary average flow conservation condition needs to be satisfied

$$
\bar{a}_{i}^{f}+\sum_{\forall j \neq i} \mathbb{E}\left[r_{j i}^{f}(\mathbf{h})\right] \leq \sum_{\forall j \neq i} \mathbb{E}\left[r_{i j}^{f}(\mathbf{h})\right]
$$

for all $(i, f)$ such that $i \neq d(f)$.

Link layer operation: As in [8] and [6], links at the outset can be scheduled to access simultaneously but orthogonally (in time or frequency) any of the channels. Let $w_{i j}^{k}(\mathbf{h}) \in$ $[0,1]$ denote the nonnegative fraction of time that link $(i, j)$ is scheduled to transmit over channel $k$ during the channel realization $\mathbf{h}$. Since every node interferes with all other nodes in the network, it must hold that

$$
\sum_{(i, j)} w_{i j}^{k}(\mathbf{h}) \leq 1, \quad \forall k
$$

This way, if $w_{i j}^{k}(\mathbf{h})=0.9$ and $w_{i^{\prime} j^{\prime}}^{k}(\mathbf{h})=0.1$, link $(i, j)$ transmits in $k$ during the $90 \%$ of the duration of realization $\mathbf{h}$, link $\left(i^{\prime}, j^{\prime}\right)$ during the $10 \%$, and all other links remain silent.

Physical layer operation: The resources adapted at the physical layer will be power and rate per link and channel. Specifically, $p_{i j}^{k}(\mathbf{h})$ will denote the instantaneous power transmitted over channel $k$ from node $i$ to node $j$ during the channel realization $\mathbf{h}$ if $w_{i j}^{k}(\mathbf{h})=1$. To obey spectrum mask constraints, it will be imposed that the instantaneous $p_{i j}^{k}(\mathbf{h})$ can never exceed a maximum prespecified level $\check{p}_{i j}^{k}$. On the other hand, the maximum average power node $i$ can transmit will be also bounded by $\check{p}_{i}$; hence,

$$
\mathbb{E}\left[\sum_{k} \sum_{j \neq i} w_{i j}^{k}(\mathbf{h}) p_{i j}^{k}(\mathbf{h})\right] \leq \check{p}_{i}, \quad \forall i
$$

Under bit error rate or capacity constraints, instantaneous rate and power variables are coupled. This rate-power coupling will be represented by the function $C_{i j}^{k}\left(\mathbf{h}, p_{i j}^{k}(\mathbf{h})\right)$. Throughout this paper it is assumed that the rate-power function $C_{i j}^{k}\left(\mathbf{h}, p_{i j}^{k}(\mathbf{h})\right)$ is increasing and strictly concave. For instance, if strong coding schemes are used, $C_{i j}^{k}\left(\mathbf{h}, p_{i j}^{k}(\mathbf{h})\right)$ is given by Shannon's capacity formula $\log \left(1+h_{i j}^{k} p_{i j}^{k}(\mathbf{h})\right)$, which is certainly increasing and strictly concave.

Problem formulation: The resource allocation algorithm will be designed so that higher exogenous average arrival rates are promoted. To this end, we will consider utility functions
$U_{i}^{f}(\cdot)$ that are strictly concave and increasing. Different flows $f$ may (and in general will) entail different utility functions. Note also that the optimization over $\bar{a}_{i}^{f}$ amounts to a flowcontrol mechanism, which is typically carried out at transport layer. Under all previous considerations, the optimal channel adaptive cross-layer resource allocation will be obtained as the solution of the following optimization problem:

$$
\begin{gathered}
\min _{\substack{\bar{a}_{i}^{f}, r_{i j}^{f}(\mathbf{h}), w_{i j}^{k}(\mathbf{h}), p_{i j}^{k}(\mathbf{h})}}-\sum_{(i, f)} U_{i}^{f}\left(\bar{a}_{i}^{f}\right) \\
\text { s. to : }(1),(2),(3), \text { and } \\
\sum_{f} r_{i j}^{f}(\mathbf{h}) \leq \sum_{k} w_{i j}^{k}(\mathbf{h}) C_{i j}^{k}\left(\mathbf{h}, p_{i j}^{k}(\mathbf{h})\right) .
\end{gathered}
$$

The cross-layer nature of the resource allocation problem is apparent because variables of different layers are jointly optimized. Note also that (4b) relates variables from different layers. The channel-adaptive nature is also apparent because among the optimization variables we have $r_{i j}^{f}(\mathbf{h}), w_{i j}^{k}(\mathbf{h})$, and $p_{i j}^{k}(\mathbf{h})$, which are all functions of $\mathbf{h}$.

\section{OPTIMUM RESOURCE ALLOCATION}

Although strictly speaking (4) is not convex, it can be trivially transformed into a convex problem (see e.g., [8] and [5], for details), which can be solved using a dual approach. Specifically, let $\rho_{i}^{f}$ and $\pi_{i}$ denote, respectively, the Lagrange multipliers associated with the average constraints in (1) and (3), and let $\boldsymbol{\lambda}$ be a vector collecting all these multipliers. Furthermore, define $\rho_{i j}^{*}:=\max _{f}\left[\rho_{i}^{f *}-\rho_{j}^{f *}\right]$ and let $\left(\dot{U}_{i}^{f}\right)^{-1}(\cdot)$ and $\left(\dot{C}_{i j}^{k}\right)^{-1}(\mathbf{h}, \cdot)$ denote, respectively, the inverse function of the derivative of $U_{i}^{f}(\cdot)$ and $C_{i j}^{k}(\mathbf{h}, \cdot)$. Based on this notation, the optimum average arrival rate and instantaneous power are given $b^{2}$

$$
\begin{aligned}
\bar{a}_{i}^{f *}(\boldsymbol{\lambda}) & =\left[\left(\dot{U}_{i}^{f}\right)^{-1}\left(\rho_{i}^{f *}\right)\right]_{0}^{\infty} \\
p_{i j}^{k *}(\mathbf{h}, \boldsymbol{\lambda}) & =\left[\left(\dot{C}_{i j}^{k}\right)^{-1}\left(\mathbf{h}, \pi_{i}^{*} / \rho_{i j}^{*}\right)\right]_{0}^{\check{p}_{i j}^{k}} .
\end{aligned}
$$

Because the instantaneous scheduling $w_{i j}^{k}(\mathbf{h})$ and routing $r_{i j}^{f}(\mathbf{h})$ variables are present only in linear constraints, finding a closedform expression for $w_{i j}^{k *}(\mathbf{h})$ and $r_{i j}^{f *}(\mathbf{h})$ is more complicated. In fact, to find $w_{i j}^{k *}(\mathbf{h})$ consider first the link functional cost

$$
\varphi_{i j}^{k}(\mathbf{h}, \boldsymbol{\lambda}):=-\rho_{i j}^{*} C_{i j}^{k}\left(\mathbf{h}, p_{i j}^{k *}(\mathbf{h}, \boldsymbol{\lambda})\right)+\pi_{i}^{*} p_{i j}^{k *}(\mathbf{h}, \boldsymbol{\lambda}) .
$$

With $\varepsilon_{W}$ representing a small positive number, the cost in (7) can be used to define the minimum link cost and the set of (sub) optimum links, respectively, as

$$
\varphi^{k *}(\mathbf{h}, \boldsymbol{\lambda}):=\min _{(i, j)} \varphi_{i j}^{k}(\mathbf{h}, \boldsymbol{\lambda})
$$

${ }^{2}$ The Karush-Kuhn-Tucker (KKT) conditions [1] associated with (4) can be used to prove the optimality of the resource allocation schemes that are presented in this section. As in the remaining of the paper, proofs are omitted due to space limitation. 


$$
\begin{aligned}
\mathcal{S}_{W}(\mathbf{h}, \boldsymbol{\lambda}, k):= & \{(i, j): \\
& \left.\varphi_{i j}^{k}(\mathbf{h}, \boldsymbol{\lambda})<\min \left\{0, \varphi^{k *}(\mathbf{h}, \boldsymbol{\lambda})+\varepsilon_{W}\right\}\right\} .
\end{aligned}
$$

Finally, based on the definitions (7)-(9),

$$
\begin{aligned}
& w_{i j}^{k *}(\mathbf{h}, \boldsymbol{\lambda})= \mathbb{1}_{\left\{(i, j) \in \mathcal{S}_{W}(\mathbf{h}, \boldsymbol{\lambda}, k)\right\}} \\
& \times \frac{\left(1-\frac{\varphi_{i j}^{k}(\mathbf{h}, \boldsymbol{\lambda})-\varphi^{k *}(\mathbf{h}, \boldsymbol{\lambda})}{\varepsilon_{W}}\right)^{2}}{\sum_{\left(i^{\prime}, j^{\prime}\right) \in \mathcal{S}_{W}(\mathbf{h}, \boldsymbol{\lambda}, k)}\left(1-\frac{\varphi_{i^{\prime} j^{\prime}}^{k}(\mathbf{h}, \boldsymbol{\lambda})-\varphi^{k *}(\mathbf{h}, \boldsymbol{\lambda})}{\varepsilon_{W}}\right)^{2}} .
\end{aligned}
$$

The optimum scheduling in (10) allows links whose associated cost is not minimum but $\varepsilon_{W}$-close to the minimum also to be scheduled for transmission, but in a proportional way: links with lower cost will transmit during more time. It is important to remark that for most channel realizations, the set $\mathcal{S}_{W}(\mathbf{h}, \boldsymbol{\lambda}, k)$ contains a single element, which amounts to saying that a single link will "win" the channel. Strictly speaking, for the cases where $\left|\mathcal{S}_{W}(\mathbf{h}, \boldsymbol{\lambda}, k)\right|>1$, the scheduling in (10) is not optimum and may incur a small penalty (always smaller than $\varepsilon_{W}$ ). However, (10) exhibits two major advantages: it is available in closed form and it is continuous with respect to $\lambda$. These will be exploited in the subsequent sections; see also [5] for a detailed derivation and justification of (10) for the case of cellular networks.

We will proceed in a similar manner to find $r_{i j}^{f *}(\mathbf{h})$. With $\varepsilon_{F}$ being a small positive number, we first define the flow cost functional, the minimum flow cost, and the (sub) optimum set of flows, respectively, as

$$
\begin{aligned}
\phi_{i j}^{f}(\boldsymbol{\lambda}) & :=\rho_{i}^{f *}-\rho_{j}^{* f}, \quad \phi_{i j}^{*}(\boldsymbol{\lambda}):=\min _{f} \phi_{i j}^{f}(\boldsymbol{\lambda}), \\
\mathcal{S}_{F}(\boldsymbol{\lambda}, i, j) & :=\left\{f: \phi_{i j}^{f}(\boldsymbol{\lambda})<\min \left\{0, \phi_{i j}^{*}(\boldsymbol{\lambda})+\varepsilon_{F}\right\}\right\} .
\end{aligned}
$$

Based on definitions (11)-(12), the optimum routing for a specific flow $f$ on link $(i, j)$ is

$$
\begin{aligned}
r_{i j}^{f *}(\mathbf{h}, \boldsymbol{\lambda}) & =C_{i j}^{*}(\mathbf{h}, \boldsymbol{\lambda}) \mathbb{1}_{\left\{f \in \mathcal{S}_{F}(\boldsymbol{\lambda}, i, j)\right\}} \\
\times & \frac{\left(1-\frac{\phi_{i j}^{f}(\boldsymbol{\lambda})-\phi_{i j}^{*}(\boldsymbol{\lambda})}{\varepsilon_{F}}\right)^{2}}{\sum_{f^{\prime} \in \mathcal{S}_{F}(\boldsymbol{\lambda}, i, j)}\left(1-\frac{\phi_{i j}^{f^{\prime}}(\boldsymbol{\lambda})-\phi_{i j}^{*}(\boldsymbol{\lambda})}{\varepsilon_{F}}\right)^{2}},
\end{aligned}
$$

where $C_{i j}^{*}(\mathbf{h}, \boldsymbol{\lambda})$ represents the optimum aggregate routing (among flows) for link $(i, j)$, which is defined as

$$
C_{i j}^{*}(\mathbf{h}, \boldsymbol{\lambda}):=\sum_{k} w_{i j}^{k *}(\mathbf{h}, \boldsymbol{\lambda}) C_{i j}^{k *}\left(\mathbf{h}, p_{i j}^{k *}(\mathbf{h}, \boldsymbol{\lambda})\right) .
$$

As before, (13) allows flows whose cost is not minimum but $\varepsilon_{F}$-close to the minimum also to be routed but in a way that flows with lower cost will route more packages.

\section{STOCHASTIC LAGRANGE MULTIPLIERS}

In the previous section, the optimal resource allocation schemes were characterized as a function of two variables: the current channel state information $\mathbf{h}$, and the optimum Lagrange multipliers $\boldsymbol{\lambda}^{*}$. If $n$ denotes the current block index (whose duration will correspond to the coherence interval of the fading channel), then for every $n$ the corresponding $\mathbf{h}[n]$ can be obtained through training. However, finding the value of $\boldsymbol{\lambda}^{*}$ is more complicated. In this paper, we will use stochastic approximation algorithms to estimate the value of the Lagrange multipliers. As a result, to implement the optimum policies, $\rho_{i}^{f *}$ and $\pi_{i}^{*}$ in (5)-(14) will be replaced by the stochastic estimates $\hat{\rho}_{i}^{f}[n]$ and $\hat{\pi}_{i}[n]$ presented next.

Let $\mu$ denote a constant stepsize and $a_{i}^{f *}[n]$ the instantaneous arrival of flow $f$ at node $i$ during block $n$ (whose expected value is $\left.\bar{a}_{i}^{f *}(\hat{\boldsymbol{\lambda}}[n])\right)$. The two following updates for the Lagrange multipliers are proposed:

$$
\begin{array}{r}
\hat{\rho}_{i}^{f}[n+1]=\left[\hat{\rho}_{i}^{f}[n]+\mu\left(a_{i}^{f}[n]+\right.\right. \\
\left.\left.\sum_{j \neq i} r_{j i}^{f *}(\mathbf{h}[n], \hat{\boldsymbol{\lambda}}[n])-\sum_{j \neq i} r_{i j}^{f *}(\mathbf{h}[n], \hat{\boldsymbol{\lambda}}[n])\right)\right]_{0}^{\infty} \\
\hat{\pi}_{i}[n+1]=\left[\hat{\pi}_{i}[n]-\mu\left(\check{p}_{i}\right.\right. \\
\left.\left.-\sum_{k} \sum_{j \neq i} w_{i j}^{k *}(\mathbf{h}[n], \hat{\boldsymbol{\lambda}}[n]) p_{i j}^{k *}(\mathbf{h}[n], \hat{\boldsymbol{\lambda}}[n])\right)\right]_{0}^{\infty} .
\end{array}
$$

Basically, (15) and (16) are stochastic versions of an epsilonsubgradient of the dual function of (4); see [1, Ch. 6] and [5] for details.

Assuming that the updates in (15) and (16) are bounded, the following result can be shown:

Proposition 1 Given $A>0$, there exists a random variable $W(\mu)$ and time instant $t$ so that

$$
\max _{n \geq t} \operatorname{Pr}\left\{\left\|\boldsymbol{\lambda}^{*}-\boldsymbol{\lambda}[n]\right\|>A\right\} \leq \operatorname{Pr}\{W(\mu)>A\}
$$

where $W(\mu) \rightarrow 0$ w.p. 1 as $\mu \rightarrow 0$.

This locking result can be proved based on the averaging approach in [7, Ch. 9]. Basically, Proposition 1 asserts that although the dual iterates do not strictly converge to the optimum value, with arbitrarily high probability they will hover within a small neighborhood of it. Together with convergence results for the dual variables, we are also interested in characterizing the convergence of the primal averages. For these primal averages, the following holds:

Proposition 2 The sample average of the stochastic primal variables converges to the ensemble average of the primal solution of (4) with probability one (w.p.1).

Proposition 2 can be proved capitalizing on the results in [3, Ch. 11], and basically guarantees the optimality of the stochastic schemes from the point of view of (4). To be more specific, consider the transmit power as an example, and let $\hat{\bar{p}}_{i}[n]:=$ $\left.n^{-1} \sum_{r=1}^{n} \sum_{k, j \neq i} w_{i j}^{k *}(\mathbf{h}[r], \hat{\boldsymbol{\lambda}}[r]) p_{i j}^{k *}(\mathbf{h}[r], \hat{\boldsymbol{\lambda}}[r])\right]$ denote the sample average of the stochastic instantaneous power allocation in (3). Remember that if the optimum values of the Lagrange multipliers were known, the ensemble average of the optimum instantaneous power at node $i$ could be found as $\bar{p}_{i}^{*}:=\mathbb{E}\left[\sum_{k, j \neq i} w_{i j}^{k *}\left(\mathbf{h}, \boldsymbol{\lambda}^{*}\right) p_{i j}^{k *}\left(\mathbf{h}, \boldsymbol{\lambda}^{*}\right)\right]$. Then, Proposition 2 establishes that $\hat{\bar{p}}_{i}[n] \rightarrow \bar{p}_{i}^{*}$ as $n \rightarrow \infty$ w.p.1. 


\section{QUEUE STABILITY AND AVERAGE DELAY}

So far the existence of queues that store packets before transmission has only been taken into account in the constraints (1). Even though the dynamics of the queues have not been explicitly considered into the formulation, it is of interest to characterize their stability as well as the delay performance of the developed resource allocation algorithms.

Let us begin by analyzing the queue dynamics. Let $q_{i}^{f}[n]$ denote the queue size for flow $f$ at node $i$, time slot $n$. Then, the queue obeys the recursion

$$
\begin{array}{r}
q_{i}^{f}[n+1]=\left[q_{i}^{f}[n]+a_{i}^{f}[n]+\right. \\
\left.\sum_{j \neq i} r_{j i}^{f *}(\mathbf{h}[n], \hat{\boldsymbol{\lambda}}[n])-\sum_{j \neq i} r_{i j}^{f *}(\mathbf{h}[n], \hat{\boldsymbol{\lambda}}[n])\right]_{0}^{\infty}
\end{array}
$$

for all $(f, i): i \neq d(f)$. In practice, arrivals and departures are magnitudes that vary with time scale smaller than $n$. This implies that definitions slightly different than the one in (18) are also possible. Such differences are not relevant for the subsequent analysis, and (18) has been chosen for mathematical simplicity. Comparing (15) to (18), it is clear that $\hat{\rho}_{i}^{f}[n]$ and $q_{i}^{f}[n]$ are related in a way that the stochastic Lagrange multipliers can be interpreted as a scaled version of the queue sizes. Specifically, if $\hat{\rho}_{i}^{f}[0]=\mu q_{i}^{f}[0]$, then it follows that $q_{i}^{f}[n]=\hat{\rho}_{i}^{f}[n] / \mu$.

The previous finding is meaningful from different points of view: i) to analyze the stability of our resource allocation algorithms; ii) to estimate the queueing delay that packets will suffer from; or iii) to establish connections with other wellknown cross-layer resource allocation algorithms (e.g., with the celebrated dynamic backpressure algorithm [2]). Next, we elaborate on i) and ii).

i) Queue stability: First of all, it is useful to analyze the stability of the stochastic resource allocation in (5)-(16). Using the fact that $q_{i}^{f}[n]=\hat{\rho}_{i}^{f}[n] / \mu$, the following result about the convergence of the (sample) average of the queues can be established:

Proposition 3 If $\hat{\bar{q}}_{i}^{f}[n]:=n^{-1} \sum_{r=1}^{n} q_{i}^{f}[r]$ denotes the sample average of the queue size $q_{i}[n]$, then

$$
\hat{\bar{q}}_{i}^{f}[n] \rightarrow \rho_{i}^{f *} / \mu \text { as } n \rightarrow \infty \quad \text { w.p. } 1 .
$$

Therefore, it holds that $\bar{q}_{i}^{f}<\infty$ as far as $\rho_{i}^{f *}<\infty$; i.e., as far as the original problem is feasible.

ii) Average delay: The relationship between queues and Lagrange multipliers can also be used to estimate the average queueing delay of the proposed stochastic resource allocation. To do so, we will use Little's result which asserts that with stable queues the average delay is given by the average aggregate queue length divided by the average aggregate arrival rate. This implies that the delay of a specific flow is $\bar{d}^{f}:=\left(\sum_{i} \bar{q}_{i}^{f}\right) /\left(\sum_{i} \bar{a}_{i}^{f}\right)$. Using the results in Proposition 3, it readily follows that the average delays for the stochastic resource algorithms developed in this paper are

$$
\bar{d}^{f}=\sum_{i} \rho_{i}^{f *} /\left(\mu \sum_{i} \bar{a}_{i}^{f *}\right), \forall f .
$$

In other words, the average delay of our stochastic algorithm can be estimated based on the optimal solution of (4), and the stepsize of the proposed iterations. Moreover, the KKT conditions can be used to show that for any node $i$ that accepts exogenous packets from flow $f$, it holds that $\rho_{i}^{f *}$ satisfies $\rho_{i}^{f *}=\dot{U}_{i}^{f}\left(\bar{a}_{i}^{f *}\right)$. Substituting the latter into (19), it follows that $\bar{d}^{f}=\sum_{i} \dot{U}_{i}^{f}\left(\bar{a}_{i}^{f *}\right) /\left(\mu \sum_{i} \bar{a}_{i}^{f *}\right)$. This means that based on the exogenous arrival rates of a given flow, the average delay for that flow can be estimated. It is worth mentioning that even in cases where the value of $\rho_{i}^{f *}$ is not available in closed form, convex optimization theory (and more specifically sensitivity analysis) can be used to decipher properties of $\rho_{i}^{f *}$ and, hence, of $\bar{d}^{f}$. $^{3}$

\section{REFERENCES}

[1] D. P. Bertsekas, Nonlinear Programming. Athena Scientific, 1999.

[2] L. Georgiadis, M. Neely, and L. Tassiulas, "Resource Allocation and Cross-Layer Control in Wireless Networks," Foundations and Trends in Networking, vol. 1, no. 1, pp. 1-144, Apr. 2006.

[3] H. J. Kushner and G. G. Yin, Stochastic Approximation Algorithms and Applications, 2nd Ed., Springer, 2003.

[4] A. G. Marques, G. B. Giannakis, and F. J. Ramos, "Stochastic resource allocation for orthogonal access based on quantized CSI: Optimality, convergence and delay analysis," in Proc. of ICASSP, Taipei, Taiwan, April 9-14, 2009.

[5] A. G. Marques, G. B. Giannakis, and J. Ramos, “Optimizing Orthogonal Multiple Access based on Quantized Channel State Information", submitted to the IEEE Trans. on Info. Theory, available at arxiv.org.

[6] A. Ribeiro and G. B. Giannakis, "Optimal FDMA over wireless fading mobile ad-hoc networks," Proc. of ICASSP, Las Vegas, Nevada, March 30-April 4, 2008.

[7] V. Solo and X. Kong, Adaptive Signal Processing Algorithms: Stability and Performance, Prentice Hall, 1995.

[8] C.Y. Wong, R.S. Cheng, K.B. Lataief, R.D. Murch, "Multiuser OFDM with adaptive subcarrier, bit, and power allocation," IEEE J. Sel. Areas Commun., vol. 17, no. 10, pp. 1747-1758, Oct. 1999.

[9] http://www.ece.umn.edu/users/antonio/RecentPublications.htm

\footnotetext{
${ }^{3}$ The views and conclusions contained in this document are those of the authors and should not be interpreted as representing the official policies, either expressed or implied, of the Army Research Laboratory or the U. S. Government.
} 